\title{
Kimura's disease of the right cheek: A case report
}

\author{
GUANGMING LUO ${ }^{1}$, FEIFEI GU ${ }^{2}$, TIANLIN LIU ${ }^{1,2}$ and YUANLIANG HUANG ${ }^{2}$ \\ ${ }^{1}$ Shanghai Engineering Research Center of Tooth Restoration and Regeneration, School of Stomatology, Tongji University, \\ Shanghai 200072; ${ }^{2}$ Department of Stomatology, Shanghai East Hospital Affiliated with Tongji University, \\ Shanghai 200120, P.R. China
}

Received October 27, 2014; Accepted October 8, 2015

DOI: 10.3892/etm.2015.2901

\begin{abstract}
Kimura's disease (KD), a chronic inflammatory disease of uncertain etiology, manifests as a painless subcutaneous swelling in the head and neck region that involves major salivary glands and regional lymph nodes. To date, the majority of cases of KD have been documented in Asian males aged 20-30 years. However, the number of reported cases of KD involving the oral and maxillofacial area is limited, and since the masses appear similar to cysts or benign tumors, the establishment of an accurate pre-operative diagnosis is challenging. The accurate diagnosis of KD is considered to require surgical excision followed by histopathological examination. In the current case, a 39-year-old man was admitted to hospital in October 2011 with a swelling evident on his right cheek. Surgical excision was performed, and histopathological observation was carried out. The formation of a lymphoid nodule accompanied by the vigorous proliferation of small blood vessels, eosinophilic infiltration and thickened cell walls were observed. No sign of recurrence of the mass has yet been observed, on the basis of the telephone follow-up interviews. These findings provide a novel insight useful in the diagnosis of KD in the oral and maxillofacial area.
\end{abstract}

\section{Introduction}

Kimura's disease (KD), a chronic inflammatory disease characterized by slowly growing subcutaneous nodules and benign reactive lymphoid proliferation in the face and neck region is predominantly observed in young Asian men (1). It is considered to be a benign disease involving subcutaneous tissues, major salivary glands and lymph nodes in the area of head and neck (2). Although numerous researchers have conducted investigations into the etiology of KD (3-5), reports concerning the underlying etiology of KD in oral and maxillofacial areas

Correspondence to: Professor Yuanliang Huang, Department of Stomatology, Shanghai East Hospital Affiliated with Tongji University, 150 Jimo Road, Shanghai 200120, P.R. China

E-mail: 13301921076@163.com

Key words: Kimura's disease, mass, treatment are limited (6). Until recently, the underlying cause of KD was unknown (7) In addition, the diagnosis of KD prior to surgery has proven challenging, since a clinical examination alone may not give an accurate diagnosis (8). In the present study, a case of KD affecting the oral and maxillofacial areas was evaluated and the corresponding pathogenesis was reported.

\section{Case report}

The patient in the present case was an otherwise healthy 39-year-old man. The patient was admitted to the Shanghai East Hospital Affiliated with Tongji University (Shanghai, China) in October 2011 with an obvious swelling on his right cheek (Fig. 1A). The patient had a 2-year history of non-painful swelling of the right-side cheek without additional symptoms. Three weeks prior to presentation, the patient sought medical attention and self-treated with an anti-bacterial agent administered orally (the name and dose of the drug are unknown). After a 3-week treatment, the mass only minimally responded to over-the-counter medication and no improvement in the patient's condition was exhibited. Therefore, the patient was referred to our hospital for further evaluation and treatment. According to the investigation conducted at the hospital, there was no history of recent febrile illness, pharyngitis, cough, rash or diarrhea and the patient had never received blood or blood products. Furthermore, the patient was not addicted to smoking or drinking. As a public servant, he had no occupational exposures and lived with his wife and daughter.

According to the results of a physical examination, the patient was in a healthy condition with a body temperature of $36.8^{\circ} \mathrm{C}$, blood pressure of $127 / 85 \mathrm{mmHg}$ and heart rate of 80 beats per minute. The swelling, located in the subcutaneous tissue, was firm and non-tender with ill-defined margins and measured $\sim 7 \times 5 \mathrm{~cm}^{2}$. The lesion was clearly observed to be both intra- and extra-oral without attachment to the right mandible. No signs of inflammation were identified in the stretched adjoining oral mucosa and overlying skin. The parotid salivary ducts were detected to be normal and the enlarged lymph nodes were identified as inconspicuous swellings in the submandibular or upper cervical regions. Surgical excision was performed and the formation of a lymphoid nodule was discovered surrounding the fibers between the endoplasm. Microscopic observation with hematoxylin and eosin staining revealed eosinophilic infiltration accompanied 

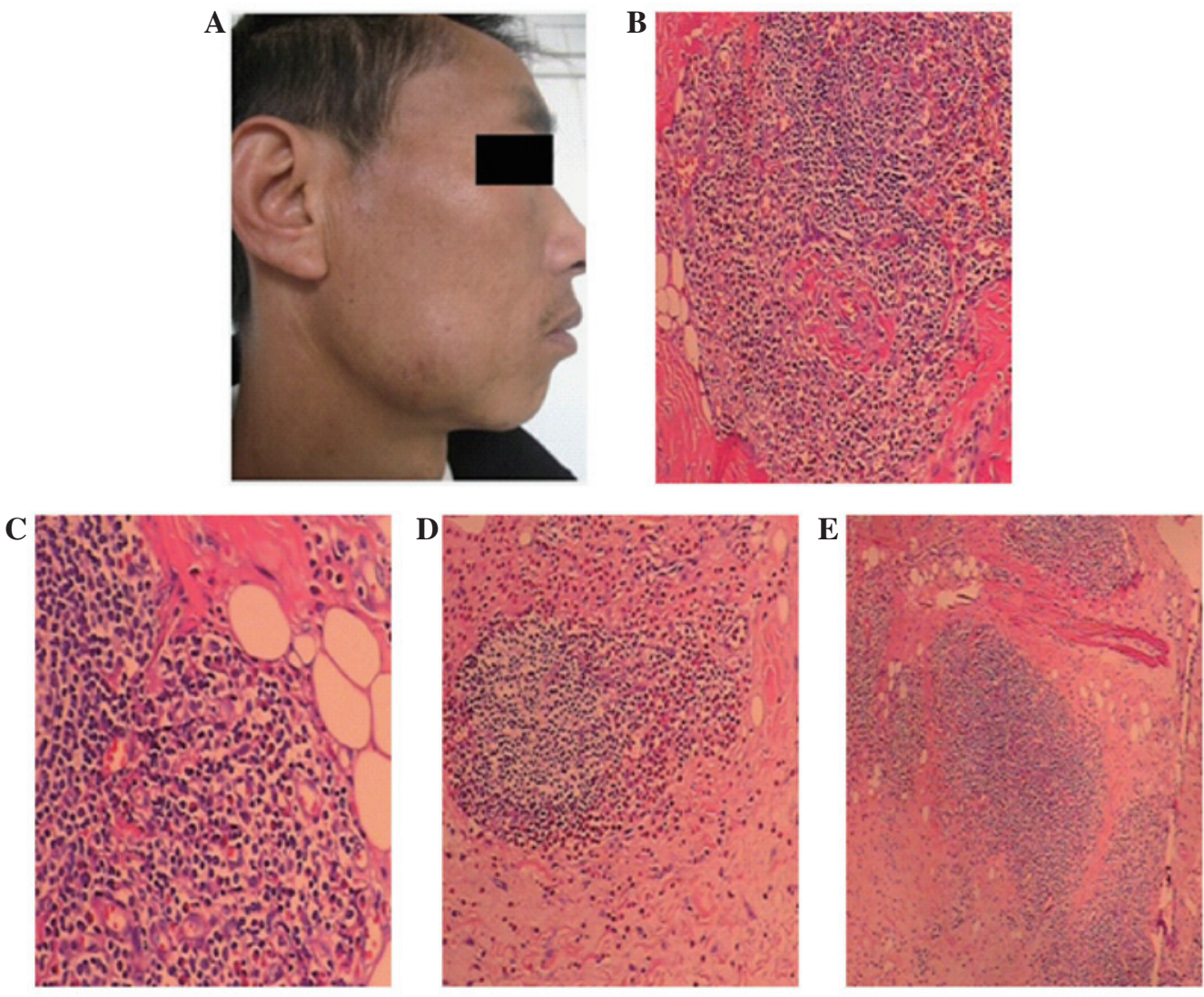

Figure 1. (A) Physical observation of the patient prior to surgery. Microscopic observation of the resected specimen revealed (B) the formation of lymphoid nodules and the proliferation of small blood vessels within the lymphoid nodules (magnification, x200), (C) the proliferation of eosinophils (magnification, $\mathrm{x} 400$ ), (D) the lymphoid nodules surrounding the fibers between the endoplasm and additional eosinophil infiltration (magnification, x200), and (E) fat and fibrous tissues exhibiting spotty lymphocytic infiltration (magnification, x100). Hematoxylin and eosin staining.

by the intense proliferation of small blood vessels and eosinophils and thickened cell walls (Fig. 1B-D). In addition, the fat and fibrous tissues exhibited spotty lymphocytic infiltration (Fig. 1E). These histopathological features provide an insight into KD in the oral and maxillofacial areas, which may serve as a reference in its diagnosis.

A follow-up interview by telephone was carried out once a year after this patient left the hospital and 3 years later, the patient reported no recurrence of the mass or subjective symptoms. Informed consent was obtained from the patient for the publication of this report.

\section{Discussion}

KD presents as a benign tumor-like lesion or cyst, which may be removed by a surgical procedure (9). The diagnosis of KD can be based on characteristic findings following surgical excision in conjunction with peripheral eosinophilia and elevated serum immunoglobulin E (IgE) levels (10). In a review of several cases, no clear conclusions were made regarding the cause of KD (11), and computed tomography and magnetic resonance imaging examinations exhibited no specific intensity or signal changes (10). Clinically, KD is typically diagnosed by oral and maxillofacial surgeons when a patient has suffered from a long history of a mass with blood eosinophilia and increased serum IgE levels, and presents with single or multiple painless subcutaneous masses (12). In addition, the confirmation of the characteristic distribution morphology with lymphadenopathy, in combination with the clinical features and laboratory examination, enables a confident preoperative diagnosis of KD (13). Despite various efforts to establish a definitive treatment for KD, none has yet been identified. The usual methods of treatment include observation, drug administration $(14,15)$, immunosuppressive therapy including tacrolimus (FK-506) (16), radiotherapy $(17,18)$, surgical excision (19) and photodynamic therapy (20). Among them, surgical excision is considered the most effective as it provides not only a treatment for $\mathrm{AD}$, but also a sample for histopathological investigations in order to facilitate an accurate diagnosis (19).

Although nonspecific, the results of histopathological analyses are considered able to eliminate the occurrence of malignancies in the differential diagnosis of KD (19). Typically, a histopathological examination is performed before a definitive diagnosis is made (21). In the present case, a histopathological examination of the diseased tissue was conducted in order to investigate the potential diagnosis of KD affecting the oral and maxillofacial areas. The examination revealed vigorous proliferation of vasculature and lymphocytic infiltration enriched with eosinophils, which may be considered as the gold standard for KD diagnosis.

The recurrence of disease is affected by various factors, including disease duration time, lesion diameter, blood eosinophil count, well-defined lesion boundaries, serum IgE levels and single or multiple lesions (22). In the current case, a single 
ill-defined lesion was exhibited, which was $>5 \mathrm{~cm}$ in diameter. However, no sign of recurrence of the mass has yet been observed on the basis of the telephone follow-up interviews, although a measurement of possible recurrence (i.e. a pathological examination) should be performed in order to further address the prognosis of the patient.

\section{References}

1. Lee $\mathbf{J}$ and Hong YS: Kimura disease complicated with bowel infarction and multiple arterial thromboses in the extremities. J Clin Rheumatol 20: 38-41, 2014.

2. Shehwaro N, Langlois AL, Gueutin V, Debchi L, Charlotte F, Rouvier P, Rottembourg J and Izzedine H: Kimura's disease: An unrecognized cause of adult-onset nephrotic syndrome with minimal change disease. Nephrol Ther 10: 46-50, 2014 (In French).

3. Reddy PKS, Prasad ALS, Sumathy TK, Shivaswamy KN and Ranganathan C: An Overlap of Angiolymphoid Hyperplasia with Eosinophilia and Kimura's Disease: Successful Treatment of Skin Lesions with Cryotherapy. Indian J Dermatol 60: 216, 2015.

4. Sah P, Kamath A, Aramanadka C and Radhakrishnan R: Kimura's disease - An unusual presentation involving subcutaneous tissue, parotid gland and lymph node. J Oral Maxillofac Pathol 17: 455-459, 2013.

5. O'Malley DP and Grimm KE: Reactive lymphadenopathies that mimic lymphoma: Entities of unknown etiology. Semin Diagn Pathol 30: 137-145, 2013.

6. Kapoor NS, O'Neill JP, Katabi N, Wong RJ and Shah JP: Kimura disease: Diagnostic challenges and clinical management. Am J Otolaryngol 33: 259-262, 2012.

7. Koh H, Kamiishi N, Chiyotani A, Takahashi H, Sudo A, Masuda Y, Shinden S, Tajima A, Kimura Y and Kimura T: Eosinophilic lung disease complicated by Kimura's disease: A case report and literature review. Intern Med 51: 3163-3167, 2012.

8. Dai L, Wei XN, Zheng DH, Mo YQ, Pessler F and Zhang BY: Effective treatment of Kimura's disease with leflunomide in combination with glucocorticoids. Clin Rheumatol 30: 859-865, 2011.
9. Shetty AK, Beaty MW, McGuirt WF Jr, Woods CR and Givner LB: Kimura's disease: A diagnostic challenge. Pediatrics 110: e39, 2002.

10. Zhang R, Ban XH, Mo YX, Lv MM, Duan XH, Shen J, Li JP, Liu XW and Xie CM: Kimura's disease: The CT and MRI characteristics in fifteen cases. Eur J Radiol 80: 489-497, 2011.

11. Punia RP, Aulakh R, Garg S, Chopra R, Mohan H and Dalal A: Kimura's disease: Clinicopathological study of eight cases. J Laryngol Otol 127: 170-174, 2013.

12. Guimaraes CS, Moulton-Levy N, Sapadin A and Vidal C: Kimura's Disease. Case Rep Med 2009: 424053, 2009.

13. Steiner D, Tudge S, Thomson P and McLean C: Kimuras disease: An unusual cause of neck mass. ANZ J Surg 76: 866-868, 2006.

14. Takeishi M, Makino Y, Nishioka H, Miyawaki T and Kurihara K: Kimura disease: Diagnostic imaging findings and surgical treatment. J Craniofac Surg 18: 1062-1067, 2007.

15. Xu X, Fu J, Fang Y and Liang L: Kimura disease in children: A case report and a summary of the literature in Chinese. J Pediatr Hematol Oncol 33: 306-311, 2011.

16. Shu DL, Ran W, GuoB, Zhang YY, Liu XZ and Liao X: Tacrolimus on Kimura's disease: A case report. Oral Surg Oral Med Oral Pathol Oral Radiol 117: e74-e78, 2014.

17. Wang Z, Zhang J, Ren Y, Dai Z, Ma H, Cui D, Su X and Song SJ: Successful treatment of recurrent Kimura's disease with radiotherapy: A case report. Int J Clin Exp Pathol 7: 45194522,2014

18. Monzen Y, Kiya K and Nishisaka T: Kimura's Disease of the Orbit Successfully Treated with Radiotherapy Alone: A Case Report. Case Rep Ophthalmol 5: 87-91, 2014.

19. Milentijeví MJ, Basić M and Petrović A: Kimura's disease in a young Balkan male. Vojnosanit Pregl 66: 66-68, 2009.

20. Abbas S, Jerjes W, Upile T, Vincent A and Hopper C: Treatment of Kimura disease with photodynamic therapy: A case study. Photodiagnosis Photodyn Ther 9: 83-86, 2012.

21. Uysal IO, Eryilmaz MA, Salk I and Abasiyanik F: Kimura disease in the parotid gland. J Craniofac Surg 22: 337-338, 2011.

22. Lin YY, Jung SM, Ko SF, Toh CH, Wong AM, Chen YR, Chan SC, Cheung YC and Ng SH: Kimura's disease: Clinical and imaging parameters for the prediction of disease recurrence. Clin Imaging 36: 272-278, 2012. 